\title{
EFFECT OF INSULIN ON LPS-INDUCED INFLAMMATORY MARKERS IN MOUSE COLON SMOOTH MUSCLE CELLS IN VITRO
}

\author{
AHMED AL-DWAIRI ${ }^{1 *}$, OTHMAN AL-SHBOUL ${ }^{1}$, MOHAMMAD ALQUDAH ${ }^{1}$, AYMAN G MUSTAFA ${ }^{2}$, \\ MAHMOUD A ALFAQIH ${ }^{1}$
}

${ }^{1}$ Department of Physiology and Biochemistry, Faculty of Medicine, Jordan University of Science and Technology, Irbid 22110, Jordan. ${ }^{2}$ Department of Anatomy, Faculty of Medicine, Jordan University of Science and Technology, Irbid 22110, Jordan. Email: Andwairi7@just.edu.jo

Received: 12 October 2017, Revised and Accepted: 18 December 2017

ABSTRACT

Objective: The aim of the research is to determine the effect of supraphysiological doses of insulin on mouse colon smooth muscle cells (CSMCs) expression and secretion of pro-inflammatory cytokines interleukin 1 alpha (IL-1 $\alpha$ ) and tumor necrosis factor alpha (TNF- $\alpha$ ) in vitro.

Methods: Freshly isolated CSMCs from BALBc mice were cultured in Dulbecco's Modified Eagle Medium and treated with various doses of insulin $(0,1,5,10$, and $20 \mathrm{nM})$ for $48 \mathrm{~h}$, with/without lipopolysaccharides (LPS; $1 \mathrm{ug} / \mathrm{mL}$ ) to induce inflammation. The levels of IL- $1 \alpha$ and TNF- $\alpha$ in the cell homogenates and conditioned media were measured using ELISA.

Results: Insulin alone $(1,5,10$, and $20 \mathrm{nM})$ did not elicit a significant change in the expression or secretion of IL-1 $\alpha$ or TNF- $\alpha$ form CSMCs; however, insulin $(10$ and $20 \mathrm{nM})$ significantly $(\mathrm{p}<0.05)$ increased the expression and secretion $(\sim 1.3-1.6$-fold) of both IL-1 $\alpha$ and TNF- $\alpha$ from CSMCs in the presence of inflammatory stimulus LPS when compared to LPS alone.

Conclusion: This study highlights the role of hyperinsulinemia on CSMC inflammation and its potential role in the pathogenesis of inflammatory bowel disease (IBD) during obesity. Measures that prevent obesity may protect against the development of IBD since the worldwide incidence of both obesity and IBD is increasing in a parallel fashion.

Keywords: Insulin, Smooth muscle, Inflammation, Inflammatory bowel disease.

(C) 2018 The Authors. Published by Innovare Academic Sciences Pvt Ltd. This is an open access article under the CC BY license (http://creativecommons. org/licenses/by/4. 0/) DOI: http://dx.doi.org/10.22159/ajpcr.2018.v11i4.23052

\section{INTRODUCTION}

Despite the important role of insulin in glucose homeostasis, lipid metabolism, growth, and energy metabolism, clinical and experimental evidence suggests that chronic hyperinsulinemia is major risk factor for the development of multiple diseases such as ischemic heart disease, stroke, atherosclerosis, cancers, Alzheimer's disease, and non-alcoholic fatty liver disease [1].

Hyperinsulinemia is usually observed in patients with obesity, type 2 diabetes mellitus, metabolic syndrome, and inflammatory bowel disease (IBD) [2-5]. The role of hyperinsulinemia in development of certain conditions such as cardiovascular diseases, stroke, and cancer is well established [1]; however, its rolein the development of IBD is notextensively investigated. The occurrence of IBD in obese patients was considered uncommon in the past [6,7]; however, recent epidemiological evidence suggests that prevalence of both obesity and IBD is increasing worldwide in a parallel fashion in the developed nations, and obesity as well as insulin resistance are relatively prevalent in IBD patients [2,3,8-11], suggesting a possible link between the altered metabolic state accompanying obesity, and the development of inflammation in the bowel. Obesity is considered a state of low-grade systemic inflammation and has been shown to be associated with altered endocrine profile, hyperinsulinemia, increased plasma leptin, decreased plasma adiponectin, elevated plasma cytokines, and immune dysregulation [12]. Obesity in IBD patients may increase the disease activity, impair drug actions, and cause post-operative surgical complications, especially in the presence of hypertrophied mesenteric adipose tissue in Crohn's disease (creeping fat) [2,9].

Conflicting data about whether insulin exerts pro-inflammatory or anti-inflammatory effects on different tissues exist [13]. Several studies showed that small doses of insulin may exert anti-inflammatory effects during euglycemic or moderately hyperglycemic conditions in the myocardium and vascular endothelial cells through the phosphoinositide 3-kinase-protein kinase B (PI3K-AKT) pathway and suppression of P-selectin and intercellular adhesion molecule 1 expression [14,15]. On the other hand, chronically elevated insulin levels during hyperglycemic conditions may exert pro-inflammatory effects through activation of mitogen-activated protein kinase (MAPK)-NF-kB signaling pathway in macrophages, hepatocytes, and vascular smooth muscle cells (SMCs), which result in stimulation of inflammatory cytokine expression [16-18].

The pathogenesis of IBD is complex and can involve multiple cytokines where they control several aspects of the inflammatory response and development of multiple extraintestinal manifestations such as arthritis, stomatitis, cholelithiasis, and hepatotoxicity $[19,20]$. Clinical and experimental evidence have shown that blockade of certain cytokines such as tumor necrosis factor (TNF- $\alpha$ ) and interleukin-1 $\alpha$ (IL-1 $\alpha$ ) by specific antibodies is effective in suppression of IBD symptoms [21-23]. The major source of cytokines in IBD is the infiltrating inflammatory cells and epithelial cells [20]; however, several studies suggest that intestinal SMCs are also greatly involved in inflammatory conditions of the bowel [24]. Clinical and experimental evidence shows that intestinal SMCs are affected structurally and functionally in IBD leading to phenotypic switching, altered contractility, and growth properties [24-26]. SMCs are capable of expression and secretion of multiple inflammatory mediators during different pathological conditions and can actively participate in IBD pathogenesis through recruitment of inflammatory cells and increased proliferation [27].

The aim of this research is to explore the effect of supraphysiological doses of insulin on the expression of inflammatory markers IL- $1 \alpha$ and 
TNF- $\alpha$ from mouse colon SMCs after exposure to inflammatory stimulus lipopolysaccharide (LPS). We hypothesize that elevated insulin levels which accompany obesity can stimulate the intestinal SMCs to produce different inflammatory mediators, thereby playing an important role in IBD pathogenesis. Measures that control obesity and prevent hyperinsulinemia can be effective in the prevention of IBD.

\section{MATERIALS AND METHODS}

\section{Materials}

Smooth muscle buffer (SMB) was prepared in-house and contained $120 \mathrm{mM} \mathrm{NaCl}, 4 \mathrm{mM} \mathrm{KCl}, 2.6 \mathrm{mM} \mathrm{KH} 2 \mathrm{PO} 4,2 \mathrm{mM} \mathrm{CaCl} 2,25 \mathrm{mM}$ HEPES (N-2-hydroxyethylpiperazine - $\mathrm{N}^{\prime} 2$ - ethanesulfonic acid), $14 \mathrm{mM}$ glucose, and $2.1 \%$ essential amino acid mixture ( $\mathrm{pH}$ was adjusted to 7.4 ). The digestion solution contained $0.5 \mathrm{mg}$ collagenase and $0.33 \mathrm{mg}$ trypsin inhibitor per $\mathrm{ml}$ of SMB. All of these chemicals were purchased from Sigma-Aldrich Inc. (St. Louis, MO, USA). Cell culture media used during the incubation process were prepared by adding $10 \%$ fetal bovine serum (FBS) (GE Healthcare, Little Chalfont, UK), $100 \mathrm{U} / \mathrm{ml}$ of penicillin, $100 \mu \mathrm{g} / \mathrm{ml}$ of streptomycin, and $2.5 \mu \mathrm{g} / \mathrm{ml}$ of amphotericin $\mathrm{B}$ to Dulbecco's Modified Eagle Medium (DMEM) with L-glutamine (Capricorn Scientific GmbH, Ebsdorfergrund, Germany). The remaining reagents were purchased from Euroclone S.P.A, (Pero, Italy). LPS was purchased from Sigma-Aldrich Inc. Human recombinant insulin was purchased from Sigma. Protein assay kit was purchased from BioRad Laboratories (Hercules, CA, USA). Specific ELISA kits for mouse TNF- $\alpha$ and IL- $1 \alpha$ were purchased from Sigma. $500 \mu \mathrm{m}$ Nitex mesh was purchased from Sigma-Aldrich Inc. (St. Louis, MO, USA).

\section{Animals}

Twenty young mature male BALB/c mice ( $\sim 12$ weeks of age, $26.5-30 \mathrm{~g})$ were provided by the animal house of Jordan University of Science and Technology. Mice were euthanized by inhalation of $\mathrm{CO}_{2}$ and the colon was rapidly excised. The colon was cut into small pieces $(2-3 \mathrm{~cm})$ in length, then placed in cold SMB with the composition described previously. All procedures were performed according to the guidelines of the Animal Care and Use Committee at Jordan University of Science and Technology.

\section{Preparation of dispersed SMCs}

Colonic SMCs were isolated by sequential enzymatic digestion of muscle strips, filtration, and centrifugation. The mucosa was scraped off with fine scissors, then the tissues were cut into thin slices $(2 \mathrm{~mm}$ long; $2 \mathrm{~mm}$ thin), and incubated for $20 \mathrm{~min}$ in SMB containing $0.5 \mathrm{mg} / \mathrm{ml}$ collagenase and $0.33 \mathrm{mg} / \mathrm{ml}$ soybean trypsin inhibitor in a shaking water bath at $31^{\circ} \mathrm{C}$. The tissues were continuously gassed with $100 \%$ oxygen during the entire isolation procedure. The partly digested tissue was washed twice with $50 \mathrm{ml}$ of collagenase-free SMB, and the muscle cells were allowed to disperse spontaneously for $10 \mathrm{~min}$ in collagenasefree medium. To eliminate broken cells and other organelles, cells were harvested by filtration through $500 \mu \mathrm{m}$ Nitex mesh and centrifuged twice at $350 \mathrm{~g}$ for $10 \mathrm{~min}$, and the process was repeated $4-5$ times. The cells were counted in a hemocytometer, and it is estimated that $95 \%$ are viable using trypan blue exclusion assay.

\section{Colon SMCs (CSMCs) proliferation assay}

Freshly isolated CSMCs were cultured in 96-well plates at $1 \times 10^{5}$ cells/well. Cells were serum deprived for $24 \mathrm{~h}$ before stimulation with either vehicle, insulin $(1 \mathrm{nM})$, insulin $(5 \mathrm{nM})$, insulin (10 nM), insulin $(20 \mathrm{nM})$, LPS $(1 \mu \mathrm{g} / \mathrm{ml})$, LPS $(1 \mu \mathrm{g} / \mathrm{ml})+$ insulin $(1 \mathrm{nM})$, LPS $(1 \mu \mathrm{g} / \mathrm{ml})+$ insulin $(5 \mathrm{nM})$, LPS $(1 \mu \mathrm{g} / \mathrm{ml})+$ insulin $(10 \mathrm{nM})$, or LPS $(1 \mu \mathrm{g} / \mathrm{ml})+$ insulin $(20 \mathrm{nM})$. Cell proliferation was assessed after $48 \mathrm{~h}$ using MTT assay (Intron Biotechnology, Korea) according to manufacturer's instructions. Briefly, CSMCs were incubated with MTT reagent (3-(4,5-dimethylthiazol-2-yl)-2,5-diphenyltetrazolium bromide) for $4 \mathrm{~h}$ at $37^{\circ} \mathrm{C}$. MTT reagent was converted to an insoluble formazan and the concentration determined by optical density at $570 \mathrm{~nm}$.

\section{Evaluation of inflammatory cytokines expression using ELISA}

Ten thousand dispersed mouse colonic SMCs ( $\mathrm{n}=3$ /group) were seeded in 6-well plate in DMEM containing 10\% FBS, $100 \mathrm{U} / \mathrm{ml}$ of penicillin,
$100 \mu \mathrm{g} / \mathrm{ml}$ streptomycin, and $2.5 \mu \mathrm{g} / \mathrm{ml}$ amphotericin B. The cells were incubated in a humidified incubator with $95 \%$ air and $5 \% \mathrm{CO}_{2}$ at $37^{\circ} \mathrm{C}$ for $48 \mathrm{~h}$ and treated with either vehicle, insulin (1 nM), insulin ( $5 \mathrm{nM})$, insulin (10 nM), insulin (20 nM), LPS (1 $\mu \mathrm{g} / \mathrm{ml})$, LPS $(1 \mu \mathrm{g} / \mathrm{ml})+$ insulin (1 nM), LPS $(1 \mu \mathrm{g} / \mathrm{ml})+$ insulin $(5 \mathrm{nM}), \operatorname{LPS}(1 \mu \mathrm{g} / \mathrm{ml})+$ insulin $(10 \mathrm{nM})$, or LPS $(1 \mu \mathrm{g} / \mathrm{ml})+$ insulin $(20 \mathrm{nM})$. After completion of the incubation period $(48 \mathrm{~h})$, the treated samples were centrifuged at $1000 \mathrm{rpm}$ for $5 \mathrm{~min}$ at $4^{\circ} \mathrm{C}$. The conditioned media were stored in $-20^{\circ} \mathrm{C}$ for further analysis, and the cells pellets were immediately lysed.

Cell lysates were prepared using BashingBeads Lysis tubes (Zymo Research, Irvine, CA, USA) and the cell lysis buffer containing protease inhibitor cocktail provided by the kit according to manufacturer's instructions. Then, lysates were centrifuged for $10 \mathrm{~min}$ at $14000 \mathrm{rpm}$ at $4^{\circ} \mathrm{C}$ and the supernatant collected for further analysis. Total protein concentration of the supernatants was measured using the DC protein assay kit. The protein concentration was then adjusted to $100 \mu \mathrm{g} / \mathrm{mL}$ in all samples.

To evaluate differential cytokine expression in the treated and control samples, specific ELISA kits for IL- $1 \alpha$ and TNF- $\alpha$ were used to measure the levels in the cell lysates and the conditioned media in the control and treated samples according to the manufacturer's protocol.

\section{Statistical analysis}

Statistical analyses were performed using GraphPad Prism 5.0 (GraphPad Software, Inc., San Diego, CA). One-way analysis of variance followed by Fisher's post hoc analysis was used to examine significant differences between groups. A $\mathrm{p}<0.05$ was considered statistically significant. All data are shown as a mean \pm standard error of the mean. Values shown are representative of three independent experiments performed in triplicates.

\section{RESULTS}

\section{Colonic SMCs identification}

Freshly isolated mouse cells were viewed at $\times 20$ objective of inverted Nikon TMS-f microscope, and they appeared spindle-shaped with varying lengths (Fig. 1a).

\section{CSMCs viability assay}

To ensure that CSMCs viability is not affected by LPS or insulin treatment, we performed an MTT assay on the treated cells. Our results indicate that cell viability is comparable between all treatment groups indicating that neither LPS nor insulin is affecting the growth of CSMCs within $48 \mathrm{~h}$ period (Fig. 1b).

\section{Assessment of cytokines expression and secretion using ELISA}

To evaluate the levels of differentially expressed cytokines in the treated samples and control, specific ELISA assays were used. Data showed that insulin alone $(1,5,10$, and $20 \mathrm{nM})$ did not elicit any change in the expression or secretion of IL- $1 \alpha$ or TNF- $\alpha$ form CSMCs when compared to control group. LPS treatment caused a marked increase in the expression of both IL- $1 \alpha$ and TNF- $\alpha(\sim 2.6$-fold increase, $p<0.01)$ when compared to control. Interestingly, insulin (10 and $20 \mathrm{nM}$ ) significantly $(p<0.05)$ increased the expression $(\sim 1.3-1.7$-fold $)$ of both IL- $1 \alpha$ and TNF- $\alpha$ from CSMCs in the presence of inflammatory stimulus LPS when compared to LPS alone (Fig. 2a and b). To further evaluate whether altered cytokine expression may affect their secretion into the media, levels of IL- $1 \alpha$ and TNF- $\alpha$ were measured in the conditioned media of treated groups. Data showed that levels of both IL- $1 \alpha$ and TNF- $\alpha$ did not change with insulin treatment $(1,5,10$, and $20 \mathrm{nM})$ alone when compared to control; however, they are significantly increased with LPS treatment $(\sim 3$-fold, $\mathrm{p}<0.05)$. Furthermore, insulin (10 and $20 \mathrm{nM})$ significantly $(\mathrm{p}<0.05)$ increased the secretion $(1.3-1.7$ fold $)$ of both IL- $1 \alpha$ and TNF- $\alpha$ from CSMCs in the presence of inflammatory stimulus LPS when compared to LPS alone (Fig. 3a and b). This data suggest that inflammatory stimuli can alter the behavior of gastrointestinal SMCs by enhancing expression of inflammatory cytokines. On the other hand, high doses of insulin can augment cytokine production in the 


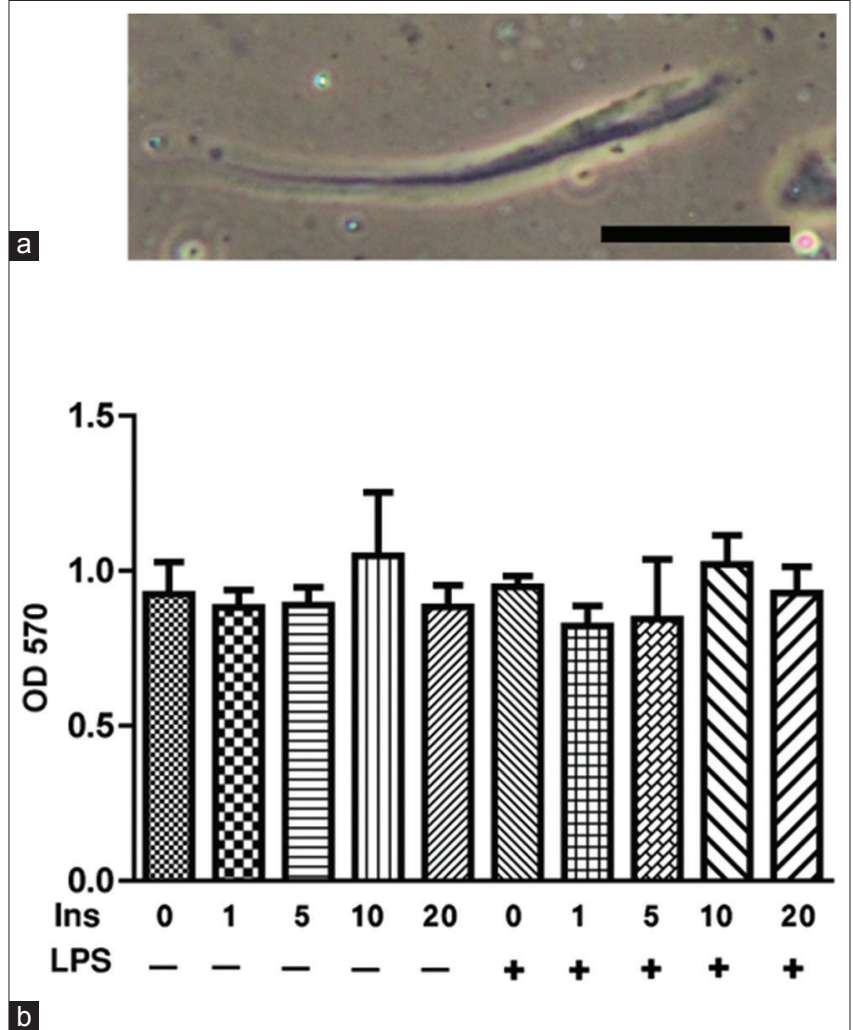

Fig. 1: (a) Isolation and identification of single mouse colon smooth muscle cells where they appeared in spindle shape under phase contrast microscopy. Cells were viewed through inverted Nikon TMS-f microscope, $\times 20$ magnification, and image captured with Canon digital camera. (b) cell viability assay for CSMCs treated with insulin (Ins) in various nanomolar doses with/ without (Lipopolysaccharide ) LPS at $1 \mu \mathrm{g} / \mathrm{mL}$ concentration

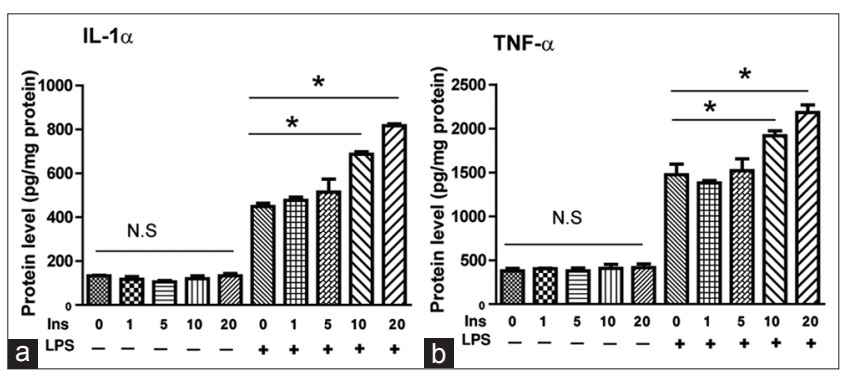

Fig. 2: Effect of insulin (Ins) treatment on the expression of inflammatory cytokines (a) interleukin and (b) tumor necrosis factor by mouse colon smooth muscle cells using ELISA in the presence or absence of inflammatory stimulus lipopolysaccharide. Cytokine levels were measured in the cellular lysate according to the manufacturer's protocol. * indicates a significant difference between groups where $p<0.05$. Bars represent mean \pm standard error of the mean

presence of inflammatory stimulus, suggesting a possible link between hyperinsulinemia and IBD pathogenesis in obese subjects.

\section{DISCUSSION}

Substantial body of evidence suggests that intestinal SMCs are affected functionally and structurally by most inflammatory conditions involving the intestine, especially IBD, leading to altered contractility, morphology, and secretion of various inflammatory mediators, thereby contributing to the pathogenesis of IBD $[24,27,28]$. IBD is a chronic disease with

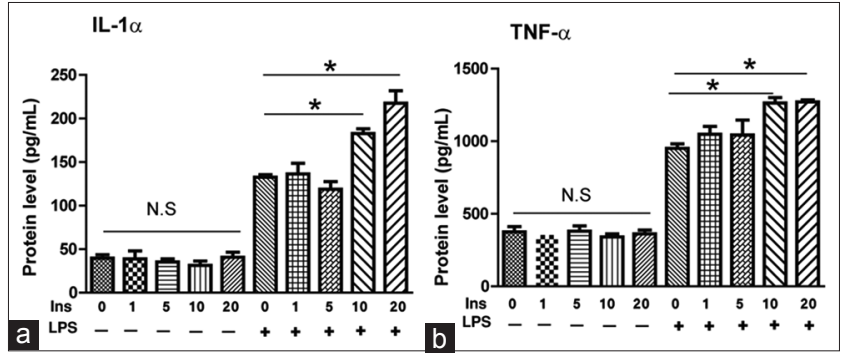

Fig. 3: Effect of insulin (Ins) treatment on the secretion of inflammatory cytokines (a) interleukin $-1 \alpha$ and (b) tumor necrosis factor- $\alpha$ by mouse colon smooth muscle cells using ELISA in the presence or absence of inflammatory stimulus lipopolysaccharide. Cytokine levels were measured in the conditioned media according to the manufacturer's protocol. *Indicates a significant difference between groups where $\mathbf{p}<0.05$

Bars represent a mean \pm standard error of the mean

multifactorial etiologies characterized by intestinal inflammation, extraintestinal symptoms, and elevated serum cytokine levels [29]. Several studies reported an increase in the prevalence of IBD in obese hyperinsulinemic patients $[2,10]$; however, the effect of insulin on development of inflammation in the bowel is not investigated. Here, we report that supraphysiological doses of insulin augment LPS-induced cytokine expression and secretion from CSMCs.

Clinical and experimental evidence suggests that hyperinsulinemia can contribute to the development of several diseases such as atherosclerosis, colon cancer, non-alcoholic steatohepatitis, and stroke [1]. However, contrasting information from different experiments show that insulin can exert both pro-inflammatory and anti-inflammatory effects in different tissues [13]. Animal experiments showed that diet rich in fructose markedly increased serum insulin levels and consequently increased reactive oxygen species, decreased nitric oxide production, and resulted in the activation of the transcription factor NF-kB and expression of downstream inflammatory genes in the cardiovascular tissues [30]. Moreover, insulin has been shown to directly induce production of different cytokines such as IL-1 $\beta$, IL-8, CCL2, and OSM in U937 macrophages by an insulin-dependent activation of NF-kB [18]. Furthermore, insulin activated pro-inflammatory gene expression through activation of PI3K independently from NF-kb in vascular SMCs [17]. In vitro studies also showed that insulin amplified LPS-induced cytokines production in THP-1 monocytes [16].

On the other hand, insulin has been reported to exert anti-inflammatory effects in multiple tissues such as cardiomyocytes, hepatocytes, and vascular endothelial and SMCs in an NF-kB-dependent manner in euglycemic or moderately hyperglycemic conditions [31]. Iwasaki et al. found that insulin exerts short-term anti-inflammatory effects mediated by PI3 Kinase-NF-kB pathway and long-term pro-inflammatory effect through activation of MAPK-NF-kB pathway in hepatocytes [13].

Our data suggest that high doses of insulin mimicking hyperinsulinemic state exerted a significant pro-inflammatory effect on mouse CSMCs in vitro in the presence of LPS and increased the expression and secretion of IL- $1 \alpha$ and TNF- $\alpha$, suggesting a pathogenic role of hyperinsulinemia in IBD development.

IL- $1 \alpha$ has a central role in innate immunity and inflammation [32]; it plays a key role in the early phases of inflammation in experimental colitis and its tissue levels correlate with the severity of the disease [33]. High levels of IL- $1 \alpha$ have been reported in colonic tissues taken from IBD patients, where it is thought to enhance recruitment of neutrophils, stimulation of IL- 6 production by macrophages, and promotion of intestinal tumorigenesis [34]. Moreover, specific blockade of $\mathrm{IL}-1 \alpha$ receptor reduces the inflammatory responses associated with experimental colitis, suggesting an essential role of IL-1 $\alpha$ in IBD 
pathogenesis [23]. Some of the effects of TNF- $\alpha$ are mediated through IL- $1 \alpha$. IL- $1 \alpha$ expression is greatly enhanced during inflammatory conditions where it initiates a cascade of event to increase inflammatory cytokines and chemokines, hence accounting for the early phases of sterile inflammation. Increased expression and secretion of both IL$1 \alpha$ and TNF- $\alpha$ together is considered an alarm cytokine signal that aggravates inflammatory responses by inducing a cascade of other proinflammatory genes [20].

TNF- $\alpha$ is potent pro-inflammatory cytokine produced as a transmembrane protein that is released as soluble cytokine by proteolytic cleavage by the metalloproteinase TNF-converting enzyme [35]. TNF- $\alpha$ is primarily secreted from activated macrophages as well as many other cell types. Several human diseases are associated with dysregulation of TNF- $\alpha$ production, and it has been reported that expression of both membrane-bound and soluble TNF- $\alpha$ by submucosal inflammatory cells is significantly increased in patients with IBD [35]. Expression of TNF- $\alpha$ in IBD stimulates different pro-inflammatory actions, such as initiation of systemic inflammatory response, increased angiogenesis, Paneth cell death, production of matrix metalloproteinase, and damage of intestinal epithelial cells [20]. Anti-TNF- $\alpha$ therapeutic antibodies such as infliximab and adalimumab are being used in the management of IBD to lessen the disease symptoms and suppress its progression, highlighting the role of TNF- $\alpha$ in the pathogenesis of IBD [21]. In our study, 10 or $20 \mathrm{mM}$ insulin enhanced production of TNF- $\alpha$ from CSMCs in the presence of inflammatory stimulus LPS, suggesting an important role for hyperinsulinemia on CSMCs-driven TNF- $\alpha$ expression in the pathogenesis of gastrointestinal inflammatory diseases possibly through recruitment and activation of different inflammatory cells.

\section{CONCLUSION}

Although the CSMCs are important in the pathogenesis of IBD, their role is rarely explored compared to the role of inflammatory and epithelial cells. Their secretory functions are also overlooked since most studies focus on the morphological changes and altered contractility that occurs during colonic inflammation. Therefore, drugs and lifestyle changes that prevent obesity and hyperinsulinemia may confer great benefits in preventing IBD, through inhibition of intestinal smooth muscle's expression and secretion of inflammatory mediators.

\section{AUTHOR CONTRIBUTIONS STATEMENT}

ANA: Conception and design of the study, acquisition of data, analysis and interpretation of data, drafting the manuscript, revising the manuscript critically for important intellectual content. OAA, MAA, AGM, and MA: Analysis and interpretation of data, revising the manuscript critically for important intellectual content.

\section{CONFLICTS OF INTEREST}

The authors declare that there are no conflicts of interest.

\section{ACKNOWLEDGMENTS}

This study was funded by the deanship of research, Jordan University of Science and Technology, Irbid, Jordan. [Grant number 20140286]. We thank Ahmad Kiwan for his valuable assistance in performing ELISA assays.

\section{REFERENCES}

1. Crofts CA. Hyperinsulinemia: A unifying theory of chronic disease? Diabesity 2015;1:34-43.

2. Singh S, Dulai PS, Zarrinpar A, Ramamoorthy S, Sandborn WJ. Obesity in IBD: Epidemiology, pathogenesis, disease course and treatment outcomes. Nat Rev Gastroenterol Hepatol 2017;14:110-21.

3. Bregenzer N, Hartmann A, Strauch U, Schölmerich J, Andus T, Bollheimer LC, et al. Increased insulin resistance and beta cell activity in patients with Crohn's disease. Inflamm Bowel Dis 2006;12:53-6.

4. Nerkar D, Mukherjee A, Mehta BK, Banerjee S. Metabolic syndrome associated complications. Int J Pharm Pharm Sci 2015;4:22-5.
5. Bollapragada MK, Shantaram M, Kumar RS. Obesity: Development, epidemiology, factors affecting, quantity, health hazards, management and natural treatment - A review. Int J Pharm Pharm Sci 2017;9:12-26.

6. Harper JW, Zisman TL. Interaction of obesity and inflammatory bowel disease. World J Gastroenterol 2016;22:7868-81.

7. Baig MM, Fatima S, Fatima M, Siddiqui S, Ali SA, Ilyas MN. Prescription pattern and cost of illness (COI) of inflammatory bowel disease (IBD) in a tertiary care hospital. Int $\mathrm{J}$ Pharm Pharm Sci 2016;9:44-7.

8. Nascimento AT, Rocha R, Coqueiro FG, Santana GO, Lyra AC. Does obesity complicate inflammatory bowel diseases? J Crohns Colitis 2012;6:1041.

9. Seminerio JL, Koutroubakis IE, Ramos-Rivers C, Hashash JG, Dudekula A, Regueiro M, et al. Impact of obesity on the management and clinical course of patients with inflammatory bowel disease. Inflamm Bowel Dis 2015;21:2857-63.

10. Long MD, Crandall WV, Leibowitz IH, Duffy L, del Rosario F, Kim SC, et al. Prevalence and epidemiology of overweight and obesity in children with inflammatory bowel disease. Inflamm Bowel Dis 2011;17:2162-8.

11. Yorulmaz E, Adali G, Yorulmaz H, Ulasoglu C, Tasan G, Tuncer I, et al. Metabolic syndrome frequency in inflammatory bowel diseases. Saudi J Gastroenterol 2011;17:376-82.

12. Heymsfield SB, Wadden TA. Mechanisms, pathophysiology, and management of obesity. N Engl J Med 2017;376:254-66.

13. Iwasaki $Y$, Nishiyama $M$, Taguchi $T$, Asai $M$, Yoshida M, Kambayashi $\mathrm{M}$, et al. Insulin exhibits short-term anti-inflammatory but long-term proinflammatory effects in vitro. Mol Cell Endocrinol 2009;298:25-32

14. Perkins JM, Joy NG, Tate DB, Davis SN. Acute effects of hyperinsulinemia and hyperglycemia on vascular inflammatory biomarkers and endothelial function in overweight and obese humans. Am J Physiol Endocrinol Metab 2015;309:E168-76.

15. Sun Q, Li J, Gao F. New insights into insulin: The anti-inflammatory effect and its clinical relevance. World J Diabetes 2014;5:89-96.

16. Brundage SI, Kirilcuk NN, Lam JC, Spain DA, Zautke NA. Insulin increases the release of proinflammatory mediators. J Trauma 2008;65:367-72.

17. Sekine O, Nishio Y, Egawa K, Nakamura T, Maegawa $H$, Kashiwagi A. Insulin activates CCAAT/enhancer binding proteins and proinflammatory gene expression through the phosphatidylinositol 3-kinase pathway in vascular smooth muscle cells. J Biol Chem 2002;277:36631-9.

18. Manowsky J, Camargo RG, Kipp AP, Henkel J, Püschel GP. Insulin-induced cytokine production in macrophages causes insulin resistance in hepatocytes. Am J Physiol Endocrinol Metab 2016;310:E938-46.

19. Guan Q, Zhang J. Recent advances: The imbalance of cytokines in the pathogenesis of inflammatory bowel disease. Mediators Inflamm 2017;2017:4810258.

20. Neurath MF. Cytokines in inflammatory bowel disease. Nat Rev Immunol 2014;14:329-42.

21. Lv R, Qiao W, Wu Z, Wang Y, Dai S, Liu Q, et al. Tumor necrosis factor alpha blocking agents as treatment for ulcerative colitis intolerant or refractory to conventional medical therapy: A meta-analysis. PLoS One 2014;9:e86692.

22. Cominelli F, Pizarro TT. Interleukin-1 and interleukin-1 receptor antagonist in inflammatory bowel disease. Aliment Pharmacol Ther 1996;10 Suppl 2:49-53.

23. Cardani D, Dusio GF, Luchini P, Sciarabba M, Solimene U, Rumio C, et al. Oral administration of interleukin-10 and anti-IL-1 antibody ameliorates experimental intestinal inflammation. Gastroenterology Res 2013;6:124-33.

24. Scirocco A, Matarrese P, Carabotti M, Ascione B, Malorni W, Severi C, et al. Cellular and molecular mechanisms of phenotypic switch in gastrointestinal smooth muscle. J Cell Physiol 2016;231:295-302.

25. Vermillion DL, Huizinga JD, Riddell RH, Collins SM. Altered small intestinal smooth muscle function in Crohn's disease. Gastroenterology 1993;104:1692-9.

26. Snape WJ Jr., Williams R, Hyman PE. Defect in colonic smooth muscle contraction in patients with ulcerative colitis. Am J Physiol 1991;261:G987-91.

27. Nair DG, Han TY, Lourenssen S, Blennerhassett MG. Proliferation modulates intestinal smooth muscle phenotype in vitro and in colitis in vivo. Am J Physiol Gastrointest Liver Physiol 2011;300:G903-13.

28. Nair DG, Miller KG, Lourenssen SR, Blennerhassett MG. Inflammatory cytokines promote growth of intestinal smooth muscle cells by induced 
expression of PDGF-r $\beta$. J Cell Mol Med 2014;18:444-54

29. Abraham C, Cho JH. Inflammatory bowel disease. N Engl J Med 2009;361:2066-78.

30. Klein AV, Kiat $\mathrm{H}$. The mechanisms underlying fructose-induced hypertension: A review. J Hypertens 2015;33:912-20.

31. Dandona P, Chaudhuri A, Mohanty P, Ghanim H. Anti-inflammatory effects of insulin. Curr Opin Clin Nutr Metab Care 2007;10:511-7.

32. Scarpa M, Kessler S, Sadler T, West G, Homer C, McDonald C, et al. The epithelial danger signal IL-1alpha is a potent activator of fibroblasts and reactivator of intestinal inflammation. Am J Pathol 2015; $185: 1624-37$
33. Papadakis KA, Targan SR. Role of cytokines in the pathogenesis of inflammatory bowel disease. Annu Rev Med 2000;51:289-98.

34. Dionne S, D'Agata DD, Hiscott J, Vanounou T, Seidman EG. Colonic explant production of IL-1 and its receptor antagonist is imbalanced in inflammatory bowel disease (IBD). Clin Exp Immunol 1998;112:435-42.

35. Brynskov J, Foegh P, Pedersen G, Ellervik C, Kirkegaard T, Bingham A, et al. Tumour necrosis factor $\alpha$ converting enzyme (TACE) activity in the colonic mucosa of patients with inflammatory bowel disease. Gut 2002;51:37-43. 